\title{
Editorial
}

\section{Gene therapy for radiation protection}

Between one-third and one-half of all cancer patients will require ionizing irradiation therapy during some point in their clinical management. With the exception of total body irradiation (TBI) used in the preparation of patients for bone marrow transplantation (BMT), radiotherapy fields are placed by the radiation oncologist with attention to the anatomic extent of normal tissues within the tumor target volume. Precise treatment planning techniques and sophisticated megavoltage linear accelerators with multi-leaf collimators are now available to shape precisely radiation fields around tumor volumes that are defined by computerized axial tomography (CAT) scan, magnetic resonance imaging (MRI) scan, positron-emission tomography (PET) scan and may conform to radiographically detectable clips positioned at the time of surgery. Despite the availability of new chemotherapeutic drugs with radiation enhancing (radiosensitizing) properties, tumor recurrence within the treatment volume in a high percentage of lung cancer patients emphasizes the fact that radiation dose escalation techniques will be required to deliver higher doses to provide a greater chance of radiocurability.

The major problem preventing the delivery of higher irradiation doses is normal tissue toxicity. The best treatment planning and beam localizing techniques and use of modern linear accelerators, which can deliver six or more radiation therapy fields to distribute the entrance dose over a wider volume of normal tissue, are all valuable. Normal tissue transit volumes still remain a significant barrier to dose escalation. Intravascular or local delivery of pharmacologic radioprotectors has been problematic, in that these agents either cannot reach the critical subcellular structures (nuclear DNA and mitochondrial membrane) involved in radiation-induced apoptosis within normal tissues, or gain access into the tumor to provide equivalent protection. Thus, there is little gain in the therapeutic ratio of tumor killing versus normal tissue damage.

Sometimes a technique which has imperfections in one clinical application is optimal in another. Such has been the potential for use of gene therapy techniques in radiation protection. The very problems which have limited gene therapy usefulness in the long-term treatment of genetic or inheritable diseases in which long-term production of specific proteins is required, have turned out to be a potential advantage for radiation therapy protection. Since the ionizing irradiation beam is only on during the treatment interval, and since prevention of nuclear DNA damage by induction of free radical oxygen and hydroxyl radicals only occurs during the time of irradiation exposure, it was reasoned that transient expression of a radioprotective transgene during the hours immediately preceding and perhaps short term after the therapeutic delivery of irradiation, might be adequate for normal tissue irradiation protection. ${ }^{1,2}$ Initial experiments carried out delivering the magnesium superoxide dismutase (MnSOD) transgene by plasmid/ liposome $^{1}$ or adenovirus ${ }^{2}$ to the lungs of mice before irradiation demonstrated a significant decrease in both the early and late effects of ionizing irradiation damage. Furthermore, long-term results of recently reported experiments demonstrate that late pulmonary induction of TGF- $\beta 1$ and TGF- $\beta 2$ messenger RNA at around 100120 days after irradiation (usually associated with the late organizing alveolitis/fibrosis effect), was also decreased by MnSOD plasmid/liposome gene therapy. ${ }^{3}$ Protection of the esophagus from acute irradiation damage by intraesophageal MnSOD plasmid/liposome delivery ${ }^{4}$ has been reported. Protection of the urinary bladder or rectum by intravesicle or intrarectal (respectively) installation of MnSOD plasmid/liposomes before irradiation appear quite feasible. In a study in progress, orthotopic Lewis lung carcinomas in the setting of intrapulmonary injection of MnSOD plasmid/liposomes demonstrated transgene expression in normal tissue but not in the tumor, and no detectable effect on irradiation killing of tumor or local control under conditions in which normal lung is protected. In each of these model systems, transgene expression in the target organ has been transient over $24-72 \mathrm{~h}$. For fractionated irradiation treatment programs of 6-8 weeks duration, plasmid/liposome administration three times per week for radiation protection during the treatment interval would be expected to be followed by clearance of the transgene from the system 3-4 days after the last treatment fraction.

There remain many unanswered but very exciting questions. Does MnSOD afford radiation protection because of its localization to the mitochondrial membrane? Clearly, copper/zinc superoxide dismutase $(\mathrm{Cu} / \mathrm{ZnSOD})$ a cytoplasmic superoxide dismutase transgene delivery, has not produced radiation protection under identical delivery conditions nor has delivery of the transgene for metallothionein. ${ }^{2}$ Radioprotective gene products, including glutathione peroxidase, catalase, gamma-glutamyltranspeptidase, and others with the capacity for induction of DNA repair remain to be tested. The positive results with MnSOD are of particular interest in view of recent data demonstrating that radiationinduced apoptosis involves critical steps at the mitochondrial membrane; including increased mitochondrial 
membrane permeability, cytochrome- $C$ release, and then activation of caspase- 3 and poly(adenosine diphosphateribose) polymerase (PARP) producing nuclear fragmentation and apoptosis. 5,6 The localization of MnSOD to the mitochondrial membrane suggests that prevention of irradiation damage in vivo, and in another study the prevention of irradiation killing of transduced cells in vitro, ${ }^{6}$ may provide an interesting model system with which to test the molecular mechanisms involved. Clinical trials for MnSOD plasmid/liposome protection of the esophagus and bladder in the setting of toxic chemoradiotherapy of lung cancer and gynecological tumors, respectively, should provide insight into the potential clinical usefulness of this technique of radioprotection. Other uses of transient expression of a transgene are also of interest. ${ }^{7}$

What other future use of radioprotective gene therapy might be appropriate outside of clinical radiotherapy? Two potential uses include environmental radioprotection and long-term protection during hibernating-stasis. There are situations in the nuclear waste cleanup industry, the management of nuclear accidents and other irradiation hazards, in which safety workers might benefit from transient systemic or specific pulmonary overexpression of a radioprotective gene(s).

Further off, but no less exciting is the potential real problem of radiation protection during prolonged stasis/hibernation. Space travel outside our solar system remains for future generations but thinking about the continual exposure of hibernating-stasis space travelers is an intriguing question for the current generation of radiation biologists. Knowledge that ionizing irradiation even at a low-dose rate of cryopreserved cells can produce genetic changes, argues that we think about development of radioprotective gene therapy for cosmic irradiation protection during space travel. Perhaps by the time of such space flights the use of germ line gene therapy will be established, and germ line introduction of transgenes with programmable activation during the years required during space travel hibernation will be available. Future space travelers may have germ line integration of radioprotective genes, perhaps activated systemically or in specific organs by externally controlled transcriptional activators, or by low temperature control during hibernation.

For the present generation of radiation oncologists and biologists, we must focus on how to translate to the clinic the transient overexpression of a radioprotective transgene which provides radiation protection during radiotherapy, and explain at the molecular level how MnSOD functions at stabilizing mitochondrial permeability, neutralizing free radicals, or both mechanisms of action.

\section{JS Greenberger Department of Radiation Oncology University of Pittsburgh School of Medicine 200 Lothrop Street, B346-PUH Pittsburgh, PA 15213, USA}

\section{References}

1 Epperly MW et al. Prevention of late effects of irradiation lung damage by manganese superoxide dismutase gene therapy. Gene Therapy 1998; 5: 196-208.

2 Epperly MW et al. Intratracheal injection of adenovirus containing the human MnSOD transgene protects athymic nude mice from irradiation-induced organizing alveolitis. Int J Radiat Oncol Biol Phys 1999; 43: 169-181.

3 Epperly MW, Travis EL, Sikora C, Greenberger JS. Magnesium superoxide dismutase (MnSOD) plasmid/liposome pulmonary radioprotective gene therapy: modulation of irradiation-induced mRNA for IL-1, TNF- $\alpha$, and TGF- $\beta$ correlates with delay of organizing alveolitis/fibrosis. Blood Bone Marrow Transplant (in press).

4 Stickle RL, Epperly MW, Greenberger JS. Prevention of irradiation-induced esophagitis by intraesophageal plasmid/ liposome delivery of the human manganese superoxide dismutase (MnSOD) transgene. Int J Radiat Oncol Biol Phys 1998; 42: 238 (Abstr. 2822).

5 Kharbanda $\mathrm{S}$ et al. Role for Bcl-xl as an inhibitor of cytosolic cytochrome-C accumulation in DNA damage-induced apoptosis. Proc Natl Acad Sci USA 1997; 94: 6939-6942.

6 Epperly MW et al. Human MnSOD transgene expression in 32D cl 3 murine hematopoietic progenitor cells protects against irradiation apoptosis through decreased caspase 3 and PARP activation. Blood 1998; 92: 197A (Abstr. 802).

7 During MJ et al. Peroral gene therapy of lactose intolerance using an adeno-associated virus vector. Nature Med 1998; 4: 1131-1135. 